practical graphs much better than most school certiticate candidates now ; secondly, the children, who are going in later on for subjects requiring mathematics, will, from thirteen or fourteen onwards, do more mathematics than the others, and, because they are segregated for part of the week, will make more rapid progress than at present.

Yours, etc., H. V. LowRy.

\title{
APPROXIMATION.
}

To the Editor of the Mathematical Gazette.

SIR,-Mr. C. V. Durell wisely prefaces his chapter on approximations in his General Arithmetic for Schools with the maxim, " The important thing about approximate answers is that they should be right as far as they go."

On page iii of the Contents in the Board of Education's Report on Curriculum and Examinations in Secondary Schools we read : "Note.-The estimated gross cost of the preparation of the Appended Report (including the

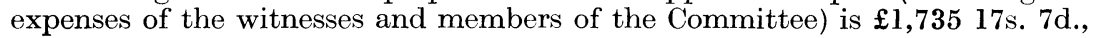
of which $£ 280$ represents the estimated cost of printing and publishing this Report.",

If this figure is correct as far as it goes, it ceases to be an estimate; if an estimate it contains false figures. No wonder there is confusion in the mind of the Fourth Former who tries to reconcile school work and the outside world.

Yours, etc., N. E. BLAKE.

\section{A GEOMETRICAL RECREATION}

\section{To the Editor of the Mathematical Gazette.}

SIR,--For a home-made near-mathematical recreation, consider the polyhedra whose vertices are the vertices of a given cuboid ; count the differentlyshaped $(a)$ polyhedra, $(b)$ tetrahedra, $(c)$ pairs of mirror images.

In a race to give the answers real mathematicians would, of course, be bunched together as the winners, but fumblers may have to bestir themselves to avoid the disgrace of being beaten by expert potato slicers. For this is also a practical man's problem : his saws and knife-blades make passable planes, and he has abundant material to his hand in wood or stone, soap, cheese or root vegetables for making cutable cuboids.

If the cutting be reckoned work, recreation must be sought in putting the pieces in place again. This, though undoubtedly an utterly infantile occupation, may give passing amusement to junior geometers, very childlike senior ones, some invalids, and even to a few mere doodlers.

It is not quite as easy as theoreticians may suppose. There is at least one carpenter who after cutting his block-under instruction-into six quadrectangular tetrahedra had to resort to the base device of matching his saw-cuts before he could get them rightly together again.

This is the most interesting variety of cutting. Indeed, if several cuboids of almost, but not quite, identical dimensions be so cut; if the surfaces be given uniform appearance and the pieces mixed; and if - the largest if of all -a real mathematician can be induced to play with them, it may happen that-despite knowing every coordinator from Feodorov to Plücker-he will not instantly reconstitute the cuboids. The puzzle is called "The Six Plopps Puzzle".

Yours, etc., O. MADDEN. 